\title{
Secondary cytotoxicity of cross-linked dermal sheep collagens during repeated exposure to human fibroblasts
}

\author{
M.J.A. van Luyn, P.B. van Wachem, L.H.H. Olde Damink*, \\ P.J. Dijkstra*, J. Feijen* and P. Nieuwenhuis \\ Department of Histology and Cell Biology, Section Biomateria/s Research, University of Groningen, \\ Oostersingel 69/2, 9713 EZ Groningen. The Netherlands; 'Department of Chemical Technology, \\ Twente University, PO Box 217, 7500 AE Enschede, The Netherlands
}

\begin{abstract}
We investigated commercially available dermal sheep collagen either cross-linked with hexamethylenediisocyanate, or cross-linked with glutaraldehyde. In previous in vitro studies we could discriminate primary, i.e. extractable, and secondary cytotoxicity, due to cell-biomaterial interactions, i.e. enzymatic actions. To develop dermal sheep collagen for clinical applications, we focused in this study on the release, e.g. elimination, of secondary cytotoxicity over time. We used the universal $7 \mathrm{~d}$ methylcellulose cell culture with human skin fibroblasts as a test system. Hexamethylenediisocyanate-cross-linked dermal sheep collagen and glutaraldehyde-crosslinked dermal sheep collagen were tested, with intervals of $6 \mathrm{~d}$, over a culture period of $42 \mathrm{~d}$. With hexamethylenediisocyanate-cross-linked dermal sheep collagen, cytotoxicity, i.e. cell growth inhibition and deviant cell morphology, was eliminated after $18 \mathrm{~d}$ of exposure. When testing glutaraldehyde-cross-linked dermal sheep collagen, the bulk of cytotoxic products was released after $6 \mathrm{~d}$, but a continuous low secondary cytotoxicity was measured up to $42 \mathrm{~d}$. As a control, non-cross-linked dermal-sheep collagen was tested over a period of $36 \mathrm{~d}$, but no secondary cytotoxic effects were observed. The differences in release of secondary cytotoxicity between hexamethylenediisocyanate-cross-linked dermal sheep collagen, glutaraldehyde-crosslinked dermal sheep collagen and non-cross-linked dermal sheep collagen are explained from differences in cross-linking agents and cross-links obtained. We hypothesize that secondary cytotoxicity results from enzymatic release of pendant molecules from hexamethylenediisocyanate-cross-linked dermal sheep collagen, e.g. formed after reaction of hydrolysis products of hexamethylenediisocyanate with dermal sheep collagen. Glutaraldehyde-crosslinked dermal sheep collagen contains residual cross-linking agents, which induce the bulk cytotoxicity. Apart from being sensitive to enzymatic degradation, glutaraldehyde-cross-linked dermal sheep collagen was also found to be sensitive to aqueous hydrolysis. Hydrolysis of cross-links may release cytotoxic products and introduce new pendant molecules within glutaraldehyde-cross-linked dermal sheep collagen, which in turn induce cytotoxicity after enzymatic attack.
\end{abstract}

Keywords: Collagen, cross-linking, fibroblasts, cytotoxicity

Received 27 January 1992; revised 4 March 1992; accepted 20 March 1992

Collagen-based materials intended for use in vivo may be cross-linked to increase their strength and persistence. However, remnants of cross-linking agents, aqueous hydrolysis of cross-links or released fragments of crosslinked collagen may induce cytotoxicity ${ }^{1-7}$. Our group investigated commercially available cross-linked dermal sheep collagen (DSC). The general aim of our studies is to obtain detailed knowledge in order to develop DSCs for clinical applications.

Correspondence to Dr M.J.A. van Luyn.
Previous in vitro studies discriminated between primary cytotoxicity, due to direct leakage of products from the materials and secondary cytotoxicity, due to release of cytotoxic products from cell-biomaterial interactions, i.e. enzymatic actions. This phenomenon was clearly observed with hexamethylenediisocyanatecross-linked DSC (HDSC) ${ }^{8,9}$, when continuous release of cytotoxic products was measured with extracted HDSC, whilst no cytotoxic products were found in their extracts. In case of glutaraldehyde-cross-linked DSC (GDSC), no clear distinction between primary and secondary cyto- 
toxicity could be made. In contrast to HDSC, two mechanisms take effect with GDSC; besides being sensitive to enzymatic actions, GDSC was also found to be sensitive to aqueous hydrolysis ${ }^{9}$.

The aim of the present study was to focus on the secondary cytotoxicity, challenging cross-linked DSCs to human fibroblasts (HF) over a longer period of time. Over a longer period, because for in vivo applications it is important to know during which period cellular interactions will release secondary cytotoxic products from DSCs and influence normal tissue ingrowth.

We used the universal methylcellulose (MC) test system $^{8-10}$. This in vitro model predicts possible cytotoxic and degradation effects of biomaterials in vivo, because both mechanisms, i.e. (limited) aqueous hydrolysis and cellular interactions, can occur in MC cell culture. Cellular interactions with HDSC, GDSC and with noncross-linked DSC (NDSC) as a control, were tested for repeated $6 \mathrm{~d}$ periods (up to $42 \mathrm{~d}$ ) by counting cell numbers and evaluating cell morphology in situ by phase-contrast light microscopy and electron microscopy.

\section{MATERIALS AND METHODS}

\section{Materials}

Human fibroblasts (established cell-line PK 84) were routinely cultured in RPMI 1640 medium (Gibco Biocult Co., Paisley, UKJ, supplemented with $10 \%$ fetal calf serum (FCS), $2 \mathrm{mM} / \mathrm{ml}$ glutamine (Glut) (Merck, Darmstadt, Germany), penicillin (Pen) and streptomycin (Strep), both 100 units $/ \mathrm{ml}$ (Gibco). The cells were incubated at $37^{\circ} \mathrm{C}$ in air containing $5 \% \mathrm{CO}_{2}$. A stock solution of methylcellulose (MC), Methocel high viscosity (3000-4000 cps) from Fluka, Bio Chemica, Buchs, Switzerland, was prepared according to Iscove and Schreier ${ }^{11}$, with Iscove's modification of Dulbecco's medium (IMDM) (ICN Biomedicals Inc., Costa Mesa, CA, USA] in a final concentration of $2.25 \%$.

Dermal sheep collagen (DSC) was ubtained from the Zuid Nederlandse Zeemlederfabriek, Oosterhout, The Netherlands. If non-cross-linked, the material is called NDSC (batch 409-018). Two types of cross-linked DSC were obtained: HDSC (batch 392-001), which was crosslinked with hexamethylenediisocyanate (HMDIC) and GDSC (batch 397-010), which was cross-linked with glutaraldehyde (GA). In short, HDSC was cross-linked for $15 \mathrm{~h}$, at $\mathrm{pH} 7.5$, with a HMDIC solution of $1.25 \%$ (Desmodur $^{\circledR}$ ) containing $0.3 \%$ of surfactant (Emulvin $\mathrm{W}^{\circledR}$ ], both obtained from Bayer, Wuppertal, Germany. GDSC was cross-linked for $17.5 \mathrm{~h}$ with a $0.5 \% \mathrm{GA}$ solution (Regulan GT $50^{\mathscr{C}}$ obtained from BASF, Basle, Switzerland), by slowly raising the $\mathrm{pH}$ from 5 to 7 . Discs with a diameter of $8 \mathrm{~mm}$ were punched from HDSC, GDSC and NDSC. The individual weights were established and all discs were sterilized by $\gamma$-irradiation, 2.5 Mrad (Gammaster, Ede, The Netherlands).

\section{Methods}

\section{Cultures}

After washing twice with phosphate-buffered saline (PBS) (NPBI, Emmer-Compascuum, The Netherlands), human skin fibroblasts (HF) were harvested from routine culture using $0.25 \%$ trypsin in $\mathrm{Ca}^{++} / \mathrm{Mg}^{++}$-free Hanks' Salt Solution (Gibco). The cells were centrifuged and resuspended in IMDM.

$\mathrm{MC} / \mathrm{HF}$ mixtures, described in detail by van Luyn et $a l^{8}$ were made by gently and thoroughly mixing HF/IMDM $(30 \%)$ with MC $(50 \%)$ and FCS $(20 \%)$. Pen, Strep and Glut had been added to IMDM in order to obtain the same final concentration in the culture gel as described for the RPMI 1640 medium. A final volume of $4.0 \mathrm{ml}$ of culture gel containing $5 \times 10^{4} \mathrm{HF}^{8}$ was placed into each well of a six well tissue culture plate [Greiner, Alphen a/d Rijn, The Netherlands) using a plastic syringe. Such cultures were either used as control culture, or, if certain discs were placed on the culture gel, as test cultures.

All cultures were incubated at $37^{\circ} \mathrm{C}$ in air containing $5 \% \mathrm{CO}_{2}$. After $24 \mathrm{~h}$, two discs of either HDSC, GDSC or NDSC, with a total weight varying between 30 and $35 \mathrm{mg}$, were put on top of the $\mathrm{MC} / \mathrm{HF}$ mixture in each well (Figure 1). After $6 \mathrm{~d}$, discs (called discs H6, G6 and N6, respectivelyl were removed from the cultures. For further cell-biomaterial interactions, these discs, first cleaned from remnants of culture gel with sterile gauze dressings, were placed on fresh cultures (again, $24 \mathrm{~h}$ after cell seeding) and incubated for a second period of $6 \mathrm{~d}$ (Figure 1). Discs from this second culture period are referred to as discs $\mathrm{H} 12, \mathrm{G} 12$ and $\mathrm{N} 12$, respectively. This culture procedure was repeated up to six times, resulting in discs $\mathrm{H} 18, \mathrm{H} 24, \mathrm{H} 30, \mathrm{H} 36, \mathrm{H} 42, \mathrm{G} 18, \mathrm{G} 24, \mathrm{G} 30, \mathrm{G} 36$, G42 and discs N18, N24, N30 and N36.

\section{Cell counts}

After every culture period discs and gel were removed. The cell layers remaining on the bottom of the wells were extensively washed three or four times with $4 \mathrm{ml}$ of PBS to remove the gel completely. The cells were then trypsinized, resuspended and counted in a Bürker counting chamber.

The cell proliferation inhibition index [CPII], expressed as a percentage of cell proliferation in control culture, was calculated as follows:

$$
\text { CPII }(\%)=100 \%-\left[\begin{array}{l}
\begin{array}{l}
\text { mean cell number of } \\
\text { culture with discs }
\end{array} \\
\begin{array}{l}
\text { mean cell number of } \\
\text { control culture }
\end{array}
\end{array} \times 100 \%\right]
$$

Cell numbers were calculated as the mean of counts \pm s.d $(n=3)$.

\section{Microscopy}

In situ photography of the cells with a phase-contrast inverted light microscope was performed after washing when the cells were covered with PBS. For electron microscopic studies, the trypsinized cells, pooled from the three wells, were washed with PBS and centrifuged. The resulting pellets were fixed with $2 \%$ GA in $0.1 \mathrm{M}$ PBS and cut into small pieces. Post-fixation was done in 1\% $\mathrm{OsO}_{4}, 1.5 \% \mathrm{~K}_{4} \mathrm{Fe}(\mathrm{CN})_{6}$ in PBS and the cells were dehydrated in graded alcohols and embedded in Epon 812. Ultrathin sections were stained with uranylacetate, dissolved in methanol and examined with a Philips 201 transmission electron microscope, operated at $40 \mathrm{kV}$. 


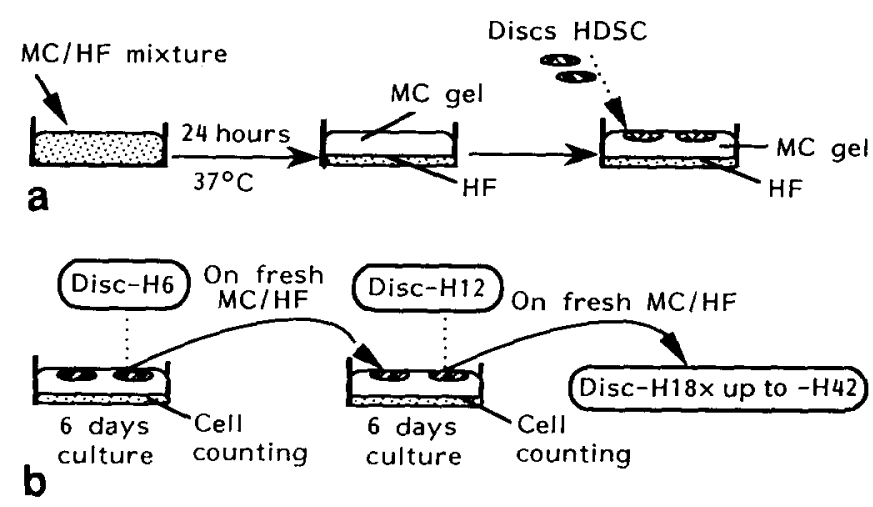

Figure 1 At $t=0,4.0 \mathrm{ml}$ of $\mathrm{MC}$ gel containing $5 \times 10^{4} \mathrm{HF}$ was placed in each well of six well tissue culture plates. After $24 \mathrm{~h}$, two discs of HDSC were placed on top of the gel and incubated for $6 \mathrm{~d}$, a. Thereafter, cells were counted and discs were transported to a fresh $24 \mathrm{~h} \mathrm{HF}$ culture. This was repeated up to $42 \mathrm{~d}$ cell culture, $b$.

\section{RESULTS}

\section{Cell counts}

HFs were seeded from $\mathrm{MC} / \mathrm{HF}$ mixtures at a density of $5 \times 10^{3} \mathrm{HF} / \mathrm{cm}^{2}$. DSC discs were placed on the MC gel of cultures after $24 \mathrm{~h}$ (Figure 1).

Cultures with discs of HDSC showed an inhibition of cell proliferation of $51.5 \pm 5.3 \%$ (called disc-H6, Figure 2a) after $6 \mathrm{~d}$. To test further effects of cell-DSC interactions, these $\mathrm{H} 6$ discs were placed for a second period of $6 \mathrm{~d}$ on a fresh HF culture. After $12 \mathrm{~d}$ of exposure, a CPII of $34 \pm 1.2 \%$ (disc-H12) was measured. The next culture period of $6 \mathrm{~d}$ resulted in a CPII of $29.2 \pm 3.8 \%$ (disc-H18). However, a fourth to a seventh culture period of $6 \mathrm{~d}$ (cultures with discs-H24, - $\mathrm{H30},-\mathrm{H} 36$ and $-\mathrm{H} 42$ ) did not show significant inhibition of cell proliferation, compared to the control culture (Figure 2a).

Discs of GDSC tested in this culture system showed a CPII of $86.9 \pm 0.6 \%$ (disc-G6, Figure $2 b$ ) after $6 \mathrm{~d}$ of culture. The second $6 \mathrm{~d}$ culture period resulted in a tremendous drop in cell-growth inhibition (CPII of $7.9 \pm 1.9 \%$ ]. Further challenging these discs, up to $42 \mathrm{~d}$, to $\mathrm{HF}$ resulted in low CPIIs, which were not significantly different from each other in disc -G12, -G18, -G24, -G30, G36, up to disc-G42 (Figure 2b).

When testing discs of NDSC, a CPII of $20.8 \pm 1.0 \%$ (disc-N6, Figure 2c) was measured after the first culture period. During further exposures, in contrast to HDSC and GDSC, no significant inhibition of cell proliferation was measured, as compared to the control. (Figure $2 \mathrm{C}$, discs-N12, -N18, -N24, -N30 and -N36.)

\section{Microscopy}

Macroscopic examinations showed cell-free zones (CFZ) upon disc removal in the cell layers of cultures with discs-H6, -G6 and also, although very small, with discsH12, $-\mathrm{H} 18$ and discs-G12 through to -G42. Light microscopic examination of the control culture showed a multilayer of well-spread cells with a few vacuole-like and sometimes with small biorefringent particles in the cytoplasm (as shown with disc-H24, Figure 3a). Cultures with discs-H6, - $\mathrm{H} 12,-\mathrm{H} 18$ and $-\mathrm{G} 6$, clearly showed decreased cell numbers, which is in agreement with the

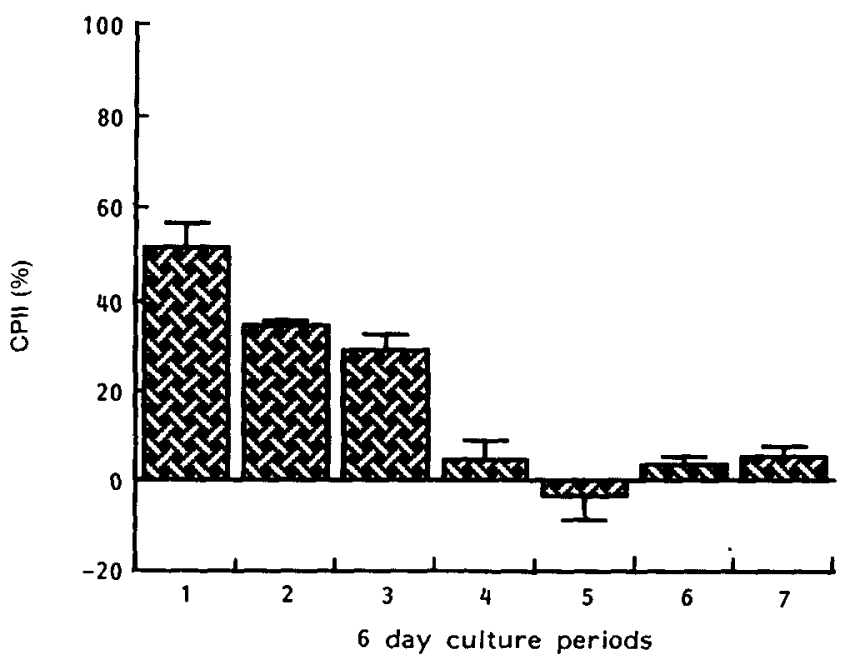

a HDSC
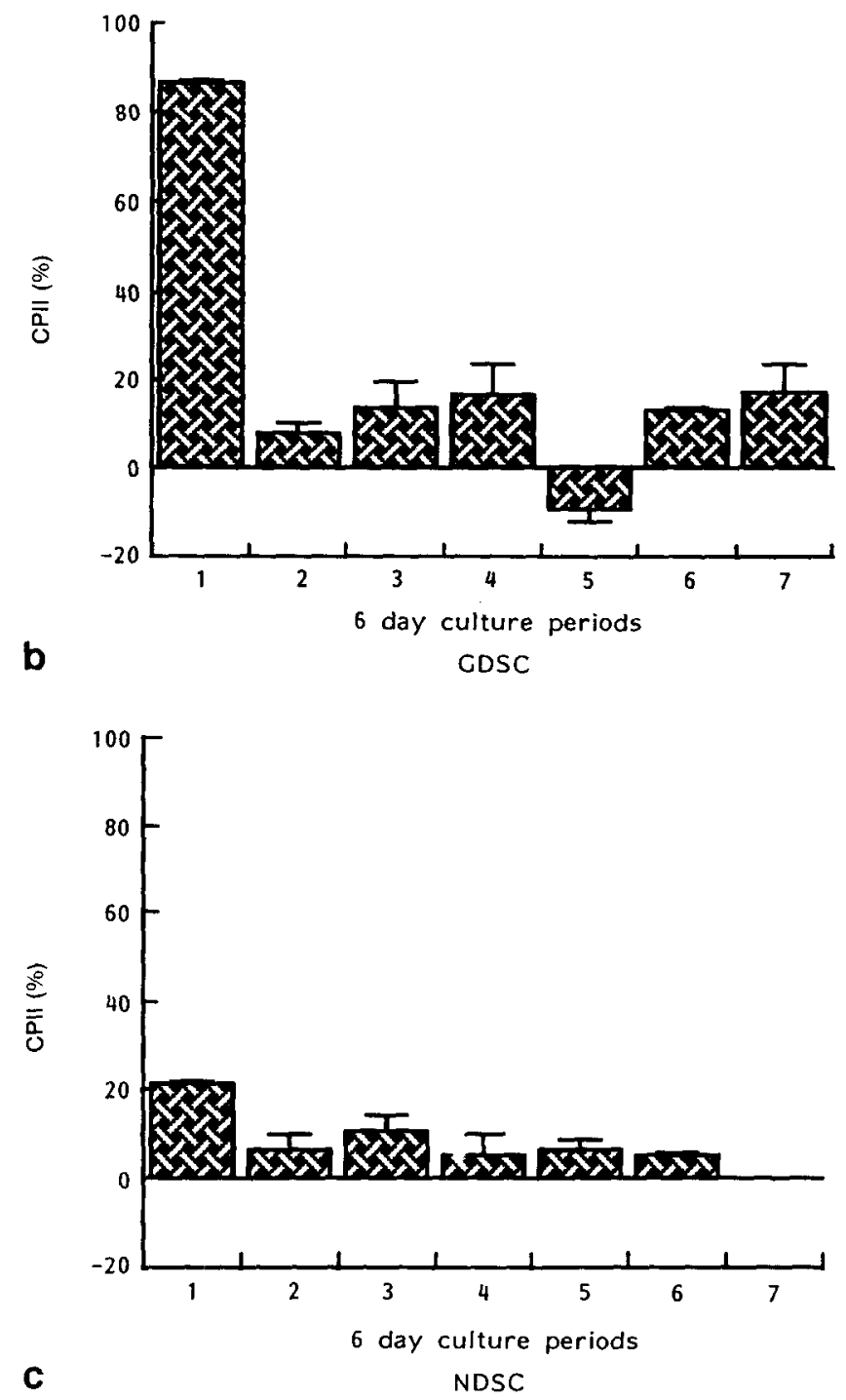

Figure 2 Cell proliferation inhibition indices (CPII) $(\%, \pm$ s.d.) of repeated culture periods. One day after cell seeding, DSC discs were repeatedly exposed to MC/HF for periods of $6 \mathrm{~d}$ (cell counting at day 7), a, cultures with discs of HDSC (discs$H 6,-H 12,-H 18,-H 24,-H 30,-H 36,-H 42) ; b$, cultures with discs of GDSC (discs-G6, -G12, -G18, -G24, -G30, -G36, -G42); c, cultures with discs of NDSC (discs-N6, -N12, $-\mathrm{N} 18,-\mathrm{N} 24$ $-\mathrm{N} 30,-\mathrm{N} 36$ ). 

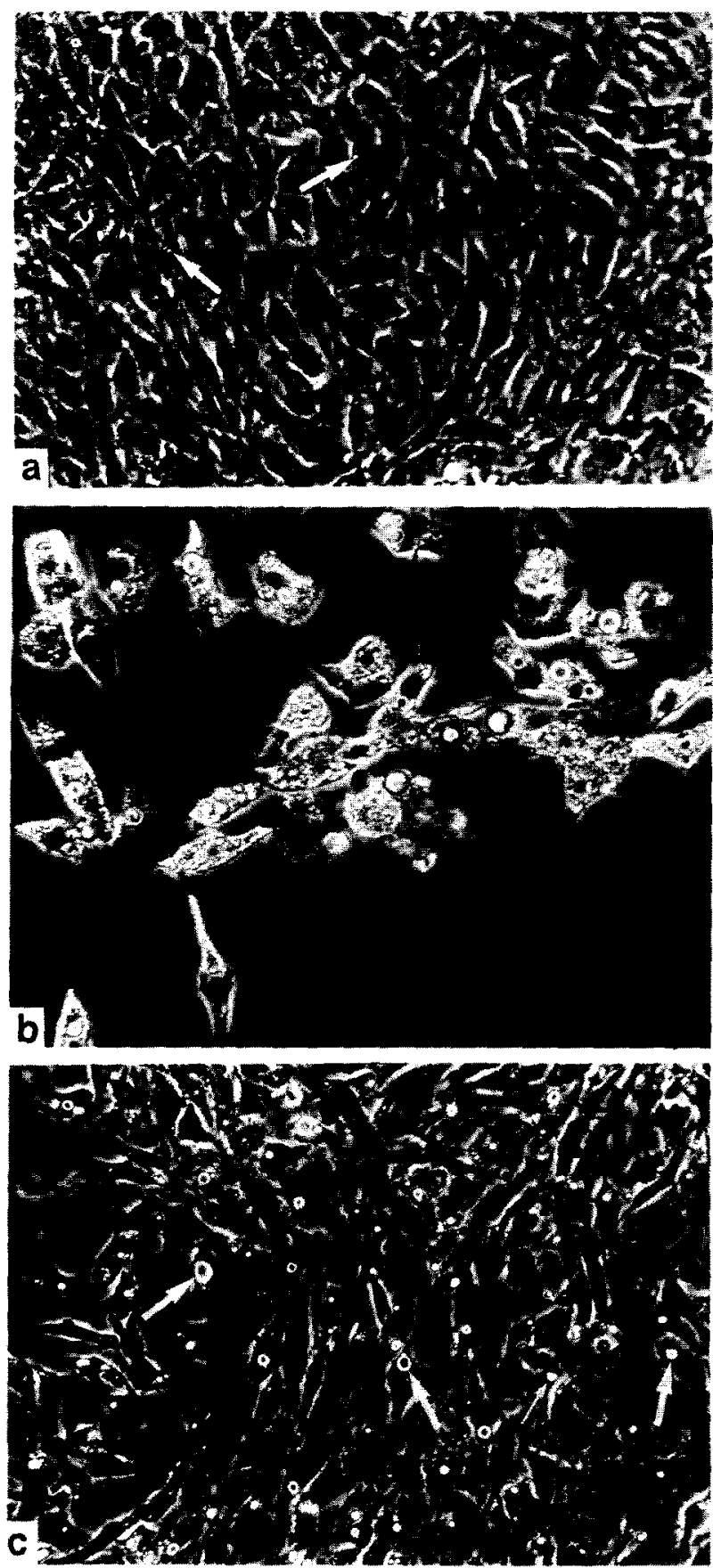

Figure 3 a, LM micrograph of a multilayer of completely spread HF cultured with disc-H24, $7 \mathrm{~d}$ after cell seeding. Some cells contain very small biorefringent particles (arrows). Original magnification $\times 720, b$. LM micrograph of HF cultured with discs-G6, $7 d$ after cell seeding. At the edge of the CFZ, decreased numbers with proportionally larger and poorly adhering cells with many biorefringent particles are found. Original magnification $\times 780$. c. LM micrograph of HF cultured with disc-G30, $7 \mathrm{~d}$ after cell seeding. Some cells poorly adhered and many cells contain biorefringent particles (arrows). Original magnification $\times 720$.

cell counts. Increased numbers of vacuole-like and biorefringent particles were observed in the cytoplasm of cells. This was more pronounced near the CFZ in cultures with discs-H6, $-\mathrm{H} 12,-\mathrm{H} 18$, but most extremely with discG6 (Figure 3b). Furthermore, proportionally larger, poorly adhering cells with elongated or spiderlike structures were observed at the edges of the CFZ.
Some of the cells of cultures with discs-G12 through to -G42 had adhered poorly and their cytoplasm contained several biorefringent particles (Figure $3 c$ ).

Although a non-confluent cell layer was observed with disc-N6, the cell morphology was similar to that of the control culture. Cell morphologies of cultures with discs-N12 through to -N36, and discs-H24 through to -H42 were also similar to the morphology of cells in the control culture (Figure 3a). Transmission electron microscopic (TEM) examination of trypsinized cells confirmed and extended the differences described above in cell morphology compared to the control culture. Previously, the biorefringent particles were found to represent lipid droplets $^{8,9}$ (Figure 4a). In our study they were found in increasing numbers in cultures with discs- $\mathrm{H} 6$ through to $-\mathrm{H} 18$, and in very high quantities in cultures with disc-G6. These cultures, with increased quantities of lipid, showed a reduction in the amount and dilatation of rough endoplasmic reticulum (RER) and, most strikingly, many cytoplasmic inclusions with remnants of dead cells. Also enlarged cells were found in these cultures. Cells of cultures with discs-G12 through to -G42 sometimes showed an increase of vacuoles and dark inclusions (myeline bodies) in the cytoplasm.

Morphologies of cultures with discs-H24 through to $-\mathrm{H} 42$, and discs-N6 through to $-\mathrm{N} 36$ were more or less similar to the morphology of the control culture.

\section{Constitution of the discs}

During $42 \mathrm{~d}$ culturing the constitution of discs of HDSC (up to disc-H42) had not changed macroscopically.

The same was found for GDSC, although by TEM, in contrast with HDSC, very small detached fragments of collagen were observed in between cells of cultures with discs-G30 through to -G42 (Figure $4 b$ ). Also collagen fragments phagocytosed by HF were observed. Sometimes they were recognized from the presence of aluminium silicate crystals, which were, as previously described $^{12,13}$, present in DSC (Figure $4 C$ ).

Macroscopically, discs of NDSC became smaller and after the first culture period of $6 d$, showed a rather smooth soap-like surface. By TEM, fragments of denatured DSC were found in between cells of cultures with discs-N12 through to $-\mathrm{N} 36$.

\section{DISCUSSION}

The aim of this study was to investigate secondary cytotoxicity, occurring as a result of interactions between (cross-linked) DSC and HF,

We used the MC cell culture previously described with $\mathrm{HF}$ as the test system ${ }^{8}$. Our results showed that:

1. Challenging HDSC to HF for three repeated $6 \mathrm{~d}$ culture periods (18 d), eliminates all cytotoxic substances.

2. Challenging GDSC to HF eliminated the bulk of cytotoxic products after $6 \mathrm{~d}$, but a continuous low secondary cytotoxicity was measured up to $42 \mathrm{~d}$.

3. Challenging NDSC for repeated culture periods to $\mathrm{HF}$ did not show release of secondary cytotoxicity. 

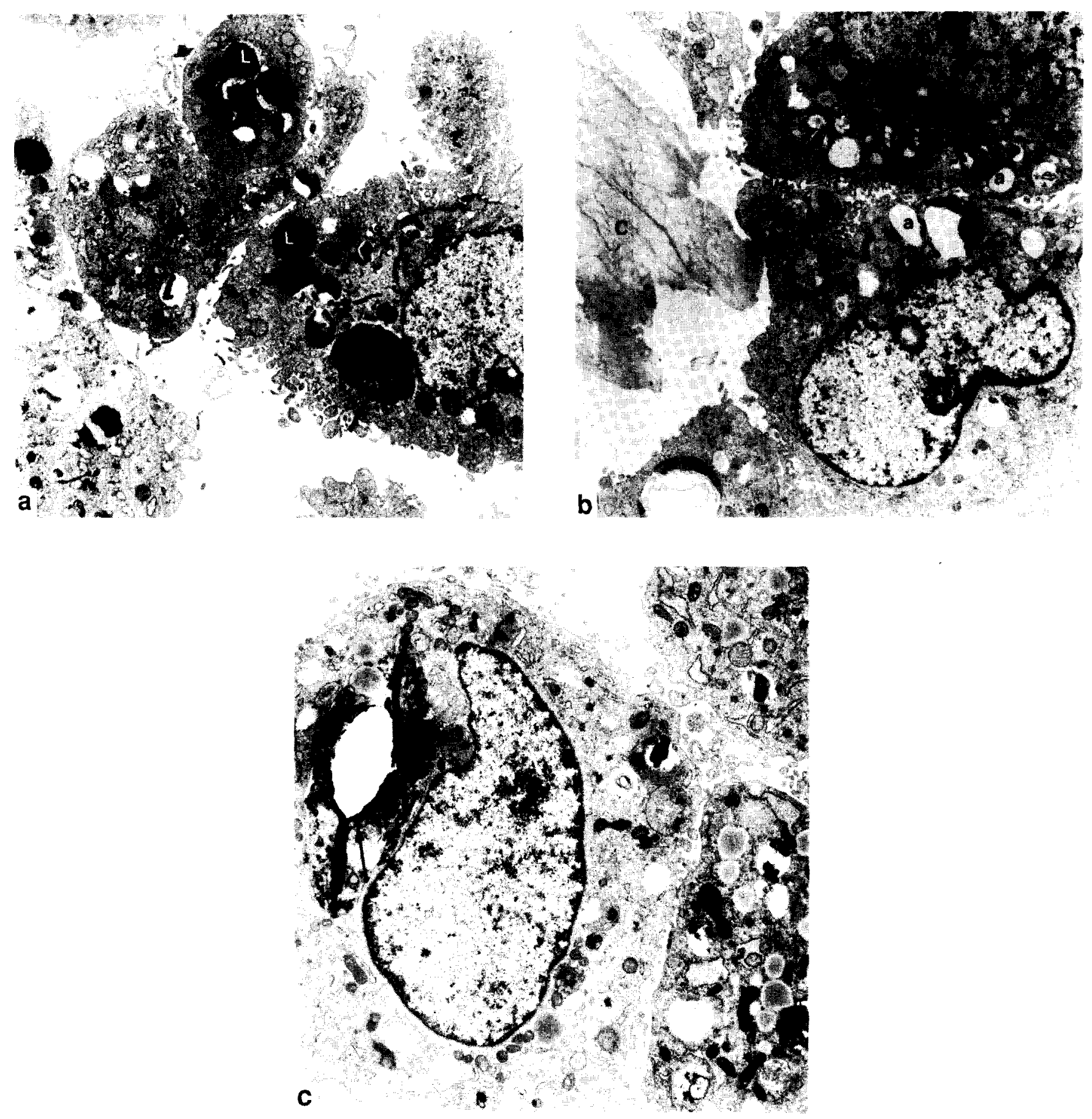

Figure 4 a, TEM micrograph of trypsinized HF cultured with disc-H18 at day 7 . Cells are in general larger and contain increased quantities of lipid droplets (L). More contracted or rounded cells, decreased presence of RER and cytoplasmic inclusions with remnants of dead cells (arrows) are observed. Original magnification $\times 7142$. b. TEM micrograph of trypsinized HF cultured with disc-G42 at day 7. Several contracted or rounded cells were observed, which contain small lipid droplets (arrows); the lipid has been (partly) removed during embedding procedure (a). Some detached collagen fragments from GDSC were observed between the cells (C). Original magnification $\times 7905$. C. TEM micrograph of trypsinized HF cultured with disc-G36 at day 7 . One cell has phagocytosed a detached fragment of GDSC. The fragment is also recognized from the presence of aluminium silicate crystals (arrow). Original magnification $\times 10752$.

Challenging HDSC to HF eliminates all cytotoxic substances within $18 \mathrm{~d}$ of cell culture

As previously discussed ${ }^{8,9}$, cross-links obtained with HMDIC are not sensitive to aqueous hydrolysis, or to enzymatic breakdown. This implies that the observed secondary cytotoxicity must result from enzymatic cleavage (e.g. by collagenase) within the collagen molecule itself. We hypothesized that the release of pendant molecules, still coupled to collagen fragments ${ }^{14}$, is responsible for the induced secondary cytotoxicity. During cross-linking, pendant molecules may have formed from reaction of the first isocyanate group of HMDIC with an $\varepsilon$-amino group of DSC, whilst a terminal $\mathrm{NH}_{2}$ group of the second isocyanate group was formed 


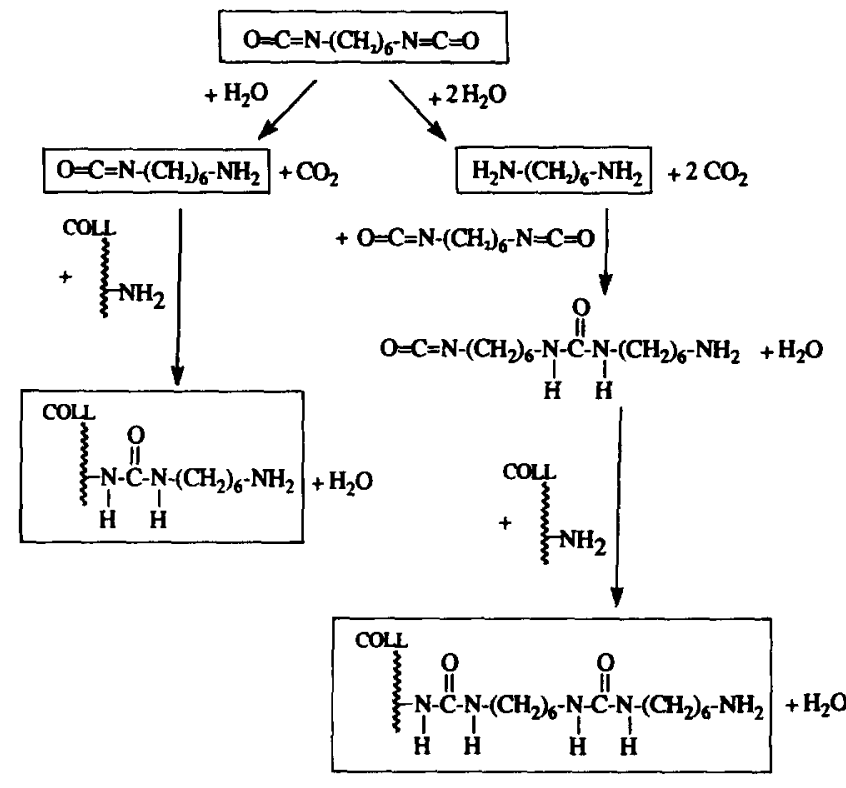

Figure 5 Formation of pendant molecules in HDSC during cross-linking. Reaction of $\mathrm{HMDIC}$ with $\mathrm{H}_{2} \mathrm{O}$ may form the hydrolysis products $\mathrm{AICH}$ and $\mathrm{DAH}$. AICH may react with $\varepsilon-\mathrm{NH}_{2}$ groups of DSC and form a pendant molecule in HDSC. Furthermore, DAH may react with HMDIC, and this product may then react with DSC and form a longed pendant molecule in HDSC.

by aqueous hydrolysis ${ }^{14}$ (Figure 5). The same pendant molecule may be formed when, as side reaction, the HMDIC hydrolysis product 1-amino-6-isocyanatehexane $(\mathrm{AICH})$ reacts with an $\varepsilon$-amino group of the collagen (Figure 5). As previously discussed ${ }^{8,9}$, the hydrolysis product 1.6-diaminohexane (DAH) may be formed and induce primary cytotoxicity. Apart from this, DAH may react with HMDIC and form another, longer pendant molecule within the collagen network (Figure 5). Statistically, enzymatic attack of the collagen molecule, e.g. by collagenase, which cleaves between the amino acids glycine and leucine ${ }^{15-18}$, will at first release the pendant molecules (by cleavage at one or two sites, Figure 6a), inducing secondary cytotoxicity. After $18 \mathrm{~d}$ HDSC is purified from cytotoxicity (Figure 2a), but enzymatic attack, and thus degradation, continues, probably releasing intact cross-links (by cleavage at two to four sites, Figure 6a), still coupled to collagen fragments. If so, these products did not induce cytotoxicity.

This hypothesis was confirmed in a pilot study, in which we incubated non-cytotoxic discs-H24 with bacterial collagenase (Sigma, St Louis, MO, USA, E C 34243,5 units $/ \mathrm{ml}$ IMDM) for $24 \mathrm{~h}$ at $37^{\circ} \mathrm{C}$ (ratio $1 \mathrm{~g}: 20 \mathrm{ml}$ ). We tested the cytotoxicity of the conditioned medium obtained and the treated disc-H24-c. In both cases, cell growth was not inhibited, and both macroscopically and by mechanical measurements clear degradation of disc-H24-c was found. Bacterial collagenase cleaves collagen at several sites ${ }^{19}$, so in this case degradation fragments with intact cross-links must have been released.

These results, as well as the early elimination of secondary cytotoxicity from HDSC, support the hypothesis of pendant molecules being responsible for secondary cytotoxicity.

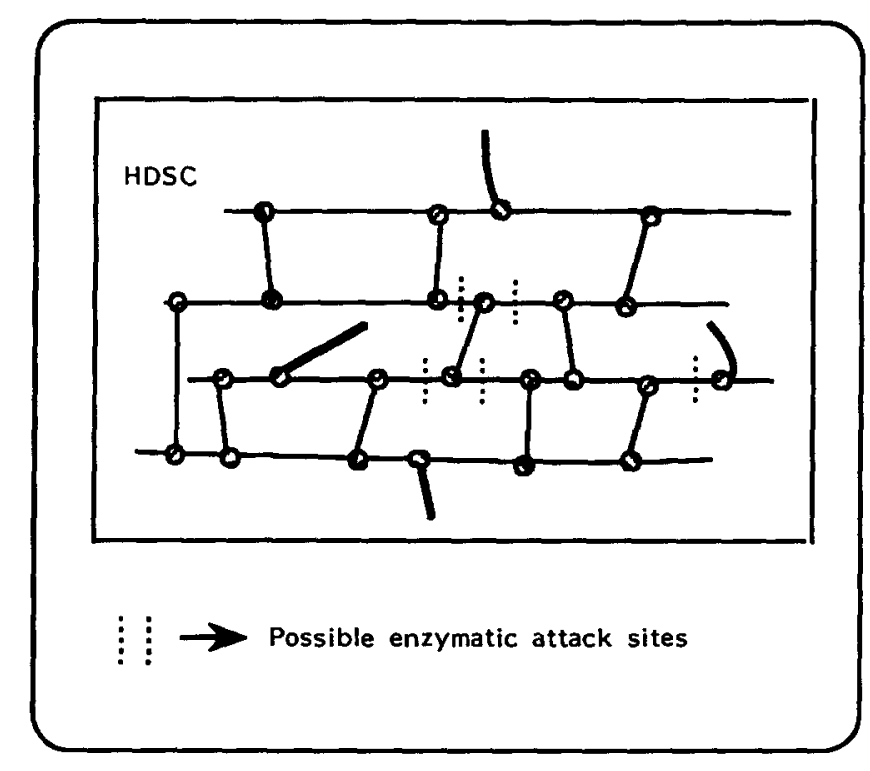

a

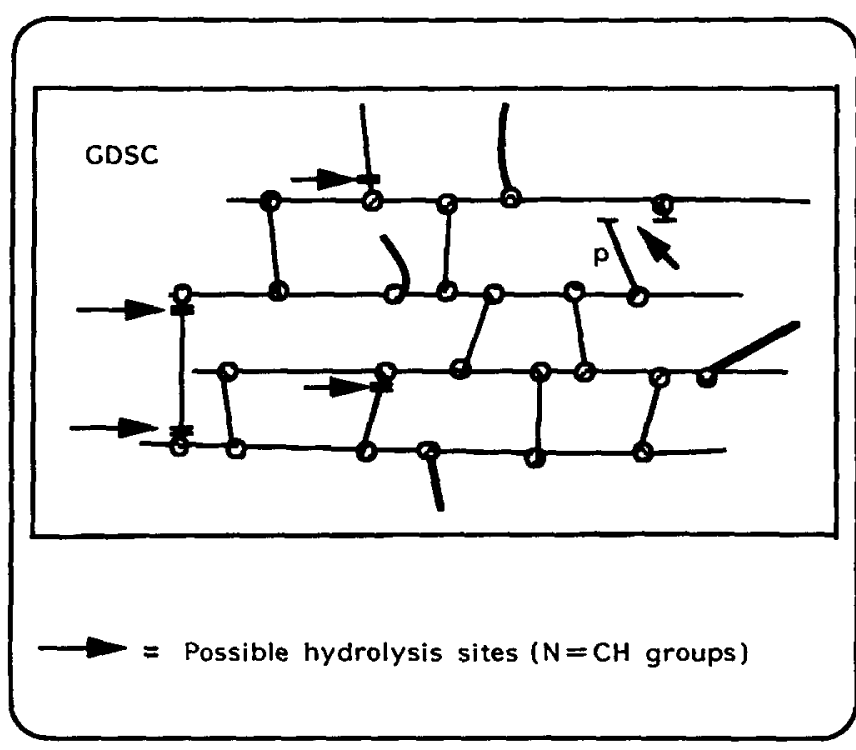

b

Figure 6 a, Scheme of HDSC network, containing HMDIC cross-links and several pendant molecules. Possible enzymatic attack sites by which pendant molecules and intact cross-links can be released are shown. b. Scheme of GDSC network with GA cross-links and pendant molecules. Possible hydrolysis sites in GA cross-links ( $\mathrm{N}=\mathrm{CH}$ groups, arrows), which may also introduce new pendant molecules in GDSC (p), are shown.

Challenging GDSC to HF eliminated the bulk of cytotoxic products after $6 \mathrm{~d}$, but a continuous low secondary cytotoxicity was measured up to $42 \mathrm{~d}$ Previously, with GDSC, no clear distinction between primary and secondary cytotoxicity could be made, because several types of cross-links of GDSC are sensitive to aqueous hydrolysis ${ }^{9}$. We previously hypothesized that aqueous hydrolysis of GDSC in the MC gel culture is limited ${ }^{9.14}$. This was confirmed in the present study, because disc-G42, in contrast to $40 \mathrm{~d}$ extracted $\mathrm{GDSC}^{9}$, had not changed into a gelatinous mass. 
After releasing residuals of GA from GDSC, probably occurring within $6 \mathrm{~d}$ (Figure $2 b$ ), other cytotoxic products can be released after aqueous hydrolytic action ${ }^{7}$ at two sites within a cross-link, or at one site within a pendant molecule (Figure $6 b$ ). The latter action will further introduce pendant molecules in GDSC. Comparable to HDSC, pendant molecules can be released by enzymatic attack, and induce secondary cytotoxicity (Figure $6 b$ ). This model may explain the continuous release of cytotoxicity from GDSC, which will probably continue up to final degradation. This also explains the higher degree of degradation of GDSC compared to HDSC, observed by TEM as very small detached fragments of collagen with discs-G30 through to -G42 (Figure 4c).

Our findings of GDSC are in agreement with in vitro studies of other investigators ${ }^{7}, 20-23$, who found low cytotoxic effects from GA-cross-linked collagens in time. Some of these authors ${ }^{21,23} \mathrm{did}$ not find confirmation for their in vitro findings in vivo. Other authors ${ }^{3.22 .24}$ observed long-term in vivo cytotoxic effects from GA-cross-linked collagens, e.g. heart valves.

Low continuing in vitro cytotoxicity may either not be effective in vivo, or the choice of animal, application site, and, even more, the microscopical evaluation level may fail to demonstrate this response. In our in vivo studies $^{12,13,25}$, after subcutaneous implantations of precultured GDSC-discs (disc-G6, Figure 2b), by use of TEM we found response for the observed low cytotoxicity of disc-G6 in vitro. With these discs, in contrast to the implanted non-toxic disc- $\mathrm{H} 24$, a low, but clear inflammatory reaction was found, after $10 \mathrm{~d}$ implantation ${ }^{25}$.

\section{Challenging NDSC for repetitive culture periods up to $36 d$ to HF did not show release of secondary cytotoxicity}

We previously reported low release of extractable primary cytotoxicity, accompanied by sensitivity for aqueous hydrolysis of NDSC ${ }^{9}$. The present study, with repeated exposures, shows that cell-related degradation of NDSC also occurs at a very high rate. This is observed macroscopically as decrease in sizes of the discs, which also had very smooth, denatured surfaces, and microscopically as denatured collagen fragments amongst cells. The results of NDSC, with respect to the degradation rate and cytotoxicity, are in agreement with our in vivo findings ${ }^{12,13,25}$. Moreover these results show, that secondary cytotoxicity is related to cross-linked DSC.

In conclusion, our series of fundamental studies, 9. 14 and the present study on dermal sheep collagen in vitro may explain possible reactions of DSCs in vivo. This shows the advantages of the MC cell culture, especially when used during repeated culture periods, in mimicking and predicting the in vivo situation.

In vivo, primary cytotoxicity of HDSC, GDSC and NDSC can be avoided by controlled washing. In case of HDSC, the remaining secondary cytotoxicity will probably soon be eliminated by cellular interactions, not seriously influencing biocompatibility over time. After elimination of the secondary cytotoxic products from HDSC, a non-toxic biocompatible scaffold will be present. In the case of washed GDSC, only low cytotoxicity will be induced up to final degradation. Whether this is a disadvantage may depend on the application area and quantity of GDSC used.
The HDSC and GDSC available commercially are not biocompatible. For future experiments, our group will focus on modifications of non-toxic cross-linked DSC e.g. cross-linking with acyl azide or carbodiimide ${ }^{26}$.

\section{REFERENCES}

1 Gendler, E., Gendler, S. and Nimni, M.E., Toxic reactions evoked by glutaraldehyde-fixed pericardium and cardiac valve tissue bioprostheses, J. Biomed. Mater. Res. 1984 18, 727

2 Bajpai, P.K. and Stull, P.K., Glutaraldehyde cross-linked porcine heart valve xenografts and cell-mediated immune response, Int. Res. Commun. Syst. Med. Sci. 1980, 8, 642

3 Speer, D.P., Chvapil, M., Eskelson, C.D. and Ulreich, J., Biological effects of residual glutaraldehyde in glutaraldehyde-tanned collagen biomaterials, J. Biomed. Mater. Res. 1980, 14, 753-764

4 Eyble, E., Griesmacher, A., Grimm, M. and Wolner, E., Toxic effects of aldehydes released from fixed pericardium on bovine aortic endothelial cells, I. Biomed. Mater. Res. 1989, 23, 1355-1365

5 Speer, D.P. and Chvapil, M., A quantitative microassay for in-vitro toxicity testing of biomaterials, J. Biomed. Mater. Res. 1981, 15, 913-922

6 Chvapil, M., Speer, D., Mora, W. and Eskelson, C., Effect of tanning agent on tissue reaction to tissuc implanted collagen sponge, J. Surg. Res. 1983, 35, 402-409

7 Hey, K.B., Lachs, C.M., Raxworthy, M.J. and Wood, E.J., Crosslinked fibrous collagen for use as a dermal implant: Control of the cytotoxic effects of glutaraldehyde and dimethylsuberimidate, Biotechnol. Appl. Biochem. 1990 , 12, 85-93

8 van Luyn, M.J.A., van Wachem, P.B., Olde Damink, L.H.H., Ten Hoopen, H., Feijen, J. and Nieuwenhuis, P., Methylcellulose culture as a new cytotoxicity test system for biomaterials, J. Mater. Sci.: Mater. Med. 1991, 2, 142-148

9 van Luyn, M.J.A., van Wachem, P.B., Olde Damink, L.H.H., Dijkstra, P.J., Feijen, J. and Nieuwenhuis, P., Relations between in vitro cytotoxicity and crosslinked dermal sheep collagens, J. Biomed. Mater. Res. 1992, 26, 1091-1110

10 van Luyn, M.J.A., van Wachem, P.B., Jonkman, M.F. and Nieuwenhuis, P., Cytotoxicity testing of wound dressings using methylcellulose cell culture, Biomaterials 1992, 13, $267-275$

11 Iscove, N.N. and Schreier, H., Clonal Growth of Cells in Semisolid or Viscous Medium, Immunological Methods Academic Press, NY, USA, 1979

12 van Wachem, P.B., van Luyn, M.J.A., Koerten, H.K., Olde Damink, L.H.H., Ten Hoopen, H., Feijen, J. and Nieuwenhuis, P., In vivo degradation of processed dermal sheep collagen cvaluated with transmission electron microscopy, Biomaterials 1991, 12, 215-223

13 van Wachem, P.B., van Luyn, M.J.A., Olde Damink, L.H.H., Feijen, J. and Nieuwenhuis, P., Tissue interactions with dermal sheep collagen implants: a transmission electron microscopical evaluation, Cells Mater. 1991, 1(3), 251-263

14 van Luyn, M.J.A., van Wachem, P.B., Olde Damink, L.H.H., Dijkstra, P.J., Feijen, J. and Nieuwenhuis, P., Enzyme-pretreatment removes cytotoxic effects of dermal sheep collagen, In, Tissue Inducing Biomaterials Symposium, Materials Research Society, 1992, 252 , 117-124

15 Murphy, G. and Reynolds, J.J., Current views of collagen degradation, progress towards understanding the resorp- 
tion of connective tissues, Bio Essays 1985, 2, 55-60 Kleiman, H.K. Klebe, R. and Martin, G. collagenous matrices in the adhesion and growth of cells, J. Cell Biol. 1981, 88, 473-485

17 Yajima, T., Acid phosphatase activity and intracellular collagen degradation by fibroblasts in vitro, Cell Tissue Res, 1986, 245, 253-260

18 Nimni, M.E. and Harkness, R.D., Molecular structure of collagen, in Collagen Vol. 1, (Ed. M.E. Nimni), CRC Press, Boca Raton, FL, USA, 3-13, 1988

19 Harris, E.D. and Krane, S.M., Medical progress, collagenases. N. Engl. I. Med. 1974, 291, 557-563

20 Huang-Lee, L.L.H., Cheung, D.T. and Nimni, M.E. Biochemical changes and cytotoxicity associated with the degradation of polymeric glutaraldehyde derived crosslinks, J. Biomed. Mater. Res. 1990, 24, 1185-1201

21 McPherson, J.M., Sawamura, S. and Armstrong, R., An examination of the biologic response to injectable, glutaraldehyde cross-linked collagen implants, I. Biomed. Mater. Kes. 1986, 20, 93-107
Eyble, E., Griesmacher, A., Grimm, M. and Wolner, E. Toxic effects of aldehydes released from fixed pericardium on bovine aortic endothelial cells, J. Biomed. Mater. Res. 1989, 23, 1355-1365

23 Law, J.K., Parsons, J.R., Silver, F.H. and Weiss, A.B., An evaluation of purified reconstituted type 1 collagen fibers, J. Biomed. Mater. Res. 1989, 23, 961-977

24 Woodroof, E.A., Use of glutaraldehyde and formaldehyde to process tissue heart valves, J. Bioeng 1978, 2, 1

25 van Wachem, P.B., van Luyn, M.J.A., Olde Damink L.H.H., Feijen, J. and Nieuwenhuis, P., In vivo interactions with (tissue culture pretreated) dermal sheep collagen, In, Tissue Inducing Biomaterials Symposium, Materials Research Society, 1992, 252, 117-124

26 Olde Damink, L.H.H., Dijkstra, P.J., van Luyn, M.J.A., van Wachem, P.B., Nieuwenhuis, P. and Feijen, I., In vitro comparison of collagen crosslinking methods, Abstract, Fourth World Biomaterials Congress, Berlin, Germany, p. 45, 1992

\section{International Conference on Materials for Biomedical Applications Tiberio Palace Hotel, Capri, Italy 6-11 June, 1993}

The conference will provide an international forum for presenting fundamental aspects of current developments and future directions for research and application of materials for biochemical applications.

\section{Conference topics}

\section{Biocompatibility \\ Biodegradation \\ Composites \\ Control release systems}

\section{Implants \\ Polymers and surface properties Regulation and standards Industrial aspects}

For further information and registration details please contact:

International Conference on Materials for Biomedical Applications, Conference Secretariat, Department of Materials and Production Engineering, Piazzale Tecchio 80, 80125 Naples, Italy.

Tel: $39817682513 / 7682400 \quad$ Fax: 39817682404 